\title{
APLICACIÓN DE LA ANTROPOMETRÍA PARA DISEÑAR EQUIPO DE PROTECCIÓN PERSONAL A TRABAJADORAS DE INDUSTRIAS MANUFACTURERAS DE COMPONENTES ELECTRÓNICOS
}

\author{
APPLICATION OF ANTHROPOMETRICS TO DESIGN PERSONAL PROTECTIVE \\ EQUIPMENT FOR FEMALE WORKERS IN ELECTRONIC COMPONENT \\ MANUFACTURING INDUSTRIES
}

\author{
Angelica María Clemente Pérez ${ }^{*}$ \\ Edith Patricia Lara Verdugo ${ }^{* *}$ \\ Eleydi Velázquez River ${ }^{* * *}$
}

\begin{abstract}
Resumen: El objetivo de este estudio es presentar un equipo de protección personal ergonómico diseñado para mujeres trabajadoras en la industria manufacturera específicamente en la inserción de componentes electrónicos. Esta investigación se realizó porque en esta área las actividades laborales se hacen de forma repetitiva por tiempos prolongados, además el método de protección personal actual utilizado son gasas colocadas de forma que envuelven los dedos. Las trabajadoras están propensas a molestias y/o lesiones en la piel de los dedos de las manos, debido a la fricción generada al insertar los componentes. Se aplicó una encuesta a 40 operadoras, de las cuales el $87 \%$ aseguró contar con al menos una lesión por esta actividad. Otro de los métodos que se utilizaron en este proyecto fue la aplicación de la antropometría; este consistió en recopilar medidas antropométricas de diferentes segmentos de la mano, como por ejemplo, largo del pulgar, largo del dedo medio, circunferencia de la muñeca entre otras, referenciadas en percentiles 5 y 95, con un nivel de confianza del 95 y desviación estándar del $5 \%$. Es importante destacar que, este proyecto estaba dividido de dos etapas; la primera consistía en presentar el prototipo del diseño de equipo de protección personal de acuerdo con los resultados obtenidos de las medidas antropométricas y encuestas realizadas. En la segunda etapa se muestra el proyecto en físico y la aceptación del diseño en el campo laboral con las trabajadoras.
\end{abstract}

Palabras clave: Antropometría, equipo de protección personal, lesiones musculoesqueléticas.

Abstract: The objective of this study is to present ergonomic personal protective equipment designed for female workers in the manufacturing industry specifically in the insertion of electronic components. This research was carried out because in this area in response to work activities carried out repeatedly for long periods of time. Currently the method used is to wrap medical gauze around the fingers. The workers are prone to discomfort and / or injuries to the skin of the fingers of the hands, due to the friction generated when inserting the components. A survey was applied to 40 operators, of which $87 \%$ claimed to have at least one injury from this activity, another method that was

\footnotetext{
"Instituto Tecnológico de Nogales. Nogales, México. Correo electrónico: angelica.cp@nogales.tecnm.mx. Orcid: https://orcid.org/oooo-0001-8756-2740. Autor de correspondencia.

**Instituto Tecnológico de Nogales. Nogales, México. Correo electrónico: Edith.lv@nogales.tecnm.mx. Orcid: https://orcid.org/oooo-0oo2-3742-3758

*** Instituto Tecnológico de Nogales. Nogales, México. Correo electrónico: Eleydi.vr@nogales.tecnm.mx. Orcid: https://orcid.org/oooo-0001-6621-267X
} 
used in this project was the application of anthropometry. This consisted of collecting anthropometric measurements of different parts of the hand, such as thumb length, middle finger length, wrist circumference, among others, referenced at $5^{\text {th }}$ and 95th percentiles, with a confidence level of 95 and standard deviation. $5 \%$. It is important to highlight that this project is divided into 2 stages, the first one only consists of presenting the prototype of the personal protective equipment design (thimbles) according to the results obtained from the anthropometric measurements and surveys carried out. In the second stage, the physical project and the acceptance of the design in the labor field with the workers will be shown.

Keywords: Anthropometry, personal protective equipment, musculoskeletal injuries.

Recepción: 29.03.2021 / Revisión: 08.04.2021 / Aceptación: 30.04.2021

\section{Introducción}

La empresa donde se hizo el estudio se encarga de ensamblar componentes electrónicos, por tal motivo, los movimientos se vuelven repetitivos y agregándole el tiempo de exposición sin equipo de protección personal, supone que generan un impacto negativo en las trabajadoras, puesto que pueden causarles lesiones físicas. Es por ello, que se pretende diseñar un guante que les ayude a reducir o eliminar cualquier lesión que pudieran presentar. "Una lesión por presión comienza con un área enrojecida en la piel, si a esta área no se le da los cuidados pertinentes se puede presentar una ampolla o costra, esto significa que el tejido se está muriendo" (Christopher \& Dana Reeve Foundation, 2016, 16 de marzo).

Las manos de un trabajador son una de las "herramientas" más importantes utilizadas en el trabajo, Sin embargo, cada año más de un cuarto de millón de personas sufren lesiones serias (y a veces discapacitantes). Al reconocer los peligros para las manos, el seguir consejos establecidos de seguridad, y el usar cubiertas protectoras u otro equipo de protección personal, los empleadores y los trabajadores pueden salvar las manos de lesiones y discapacidad. Una de las causas más serias, y al mismo tiempo más comunes, de lesiones a las manos es el usar equipo defectuoso o sin protección. Adicionalmente, los movimientos repetitivos constantes (tal como se encuentra en trabajos de ensamble) pueden causar tensión indebida en las muñecas y las manos (dedos) a menos que se tomen medidas de protección (Compañía de Producción BP, Administración de Seguridad y Salud Ocupacional, y Departamento de Seguros de Texas, 2019, 9 de diciembre).

Un artículo publicado en el 2003 en el estado de Aragua, Venezuela, sobre la investigación de accidentes con lesiones de las manos y los dedos arrojó que de los 623 accidentes reportados y declarados, 294 reportaron lesiones en las manos y los dedos de los trabajadores, lo cual representa una frecuencia de accidentes con lesiones de acuerdo con la parte afectada del 47,19\%. Estos estudios presentan información suficiente de cómo las manos en la mayoría de los países se comprometen de manera frecuente con la actividad laboral, coincidiendo con otros autores que han investigado la problemática de los accidentes laborales (Martínez, Fragiel \& Nava, 2003).

De acuerdo a NOTIMEX (2019, 12 de junio) en México se registran más de 500 mil 
accidentes de trabajo y según cifras de la Asociación Interdisciplinaria de Salud Ocupacional e Higiene en México el costo promedio de cada accidentes es de 200 mil pesos. El jefe de cirugía de mano del Hospital de Traumatología y Ortopedia Lomas verdes, del Instituto Mexicano del Seguro Social (IMSS), el doctor, Joaquín Díaz López, expresó que las manos son las extremidades que con mayor frecuencia se lesionan cuando ocurre un accidente. También comentó que el 60 por ciento de las lesiones que se atienden en dicho hospital son fracturas de falanges (huesos de los dedos) y metacarpianos (huesos de la muñeca).

Como se mencionó anteriormente, las manos son la parte del cuerpo que más se lesionan cuando ocurren accidentes en actividades laborales, sin embargo, es relevante destacar que otro factor que ponen en riesgo a las manos es la carga física de trabajo, este factor se analiza con relación a dos indicadores: el esfuerzo físico realizado por el trabajador al ejecutar su tarea, y por el otro, las molestias musculoesqueléticas que el trabajador achaca a posturas. En la demanda física del trabajo las más habituales son los movimientos repetitivos de manos y/o brazos (59\%) en actividades de la industria manufacturera, almacenamiento, conducción y transporte (Valle \& Otero, 2015).

Además de las exigencias físicas que podrían ocasionar lesiones internas, se encuentran las enfermedades cutáneas, las cuales se pueden presentar como una simple molestia o hasta incapacitar a la persona, lo cual conllevaría a un bajo desempeño que afectaría tanto al personal como a la empresa. Como lo mencionaron Wolfgang y Vedder (2000), para evitar las enfermedades de la piel relacionadas con el trabajo, hace falta adoptar medidas específicas para protegerla antes de iniciar la jornada y procurar cuidados sistemáticos al finalizar el trabajo.

De acuerdo a Alcántara (2019, 23 de mayo) los riesgos más recurrentes en las lesiones de las manos son: riesgos mecánicos de los cuales se pueden mencionar golpes, cortes, desgarres, pinchazos o atrapamiento en maquina; otro riesgo es la incomodidad y molestias como sudor, alergias y rozaduras; están presentes también los riesgos eléctricos; los riesgos físicos tales como vibraciones, radiaciones y productos radiactivos; riesgos biológicos y riesgos térmicos, como calor, frío, fuego, salpicaduras y materiales calientes.

El objetivo de este proyecto es el diseño de un equipo de protección personal ergonómico conformado por tres dedales unidos en forma de guante, con los cuales se busca evitar la fricción, la rozaduras y aliviar la presión e irritación generada por la repetitividad de la actividad y el tiempo de exposición que conlleva a este tipo de molestias y lesiones adicionales.

\section{Materiales y métodos}

De acuerdo al objetivo definido en este estudio, el método que se siguió fue un diseño no experimental, descriptivo y de corte transversal.

El estudio se realizó a 40 trabajadoras de una empresa maquiladora de ensamble de componentes electrónicos. Lo primero que se llevó a cabo fue la toma de registro fotográfico que muestran las operaciones que realizan las operadoras, con el fin de presentar el poco o 
nulo equipo de protección personal que utilizan al realizar su trabajo. Posteriormente, se aplicó un cuestionario de sintomatología para determinar la situación o estado real de las trabajadoras después de concluir su jornada laboral. Este cuestionario consta de 6 preguntas fáciles de contestar, 3 de ellas están enfocadas a si sienten malestares o si utilizan algún equipo para protegerse las manos y las otras 3 están enfocadas a la aceptación del producto por parte de ellas.

El tercer paso que se realizó en la metodología consistió en establecer un procedimiento de medición antropométrica, el cual consistió en la toma de 6 medidas de la mano, las cuales se clasificaron en A, B, C, D, E y F.

Figura 1. Segmentos de la mano que se midieron.
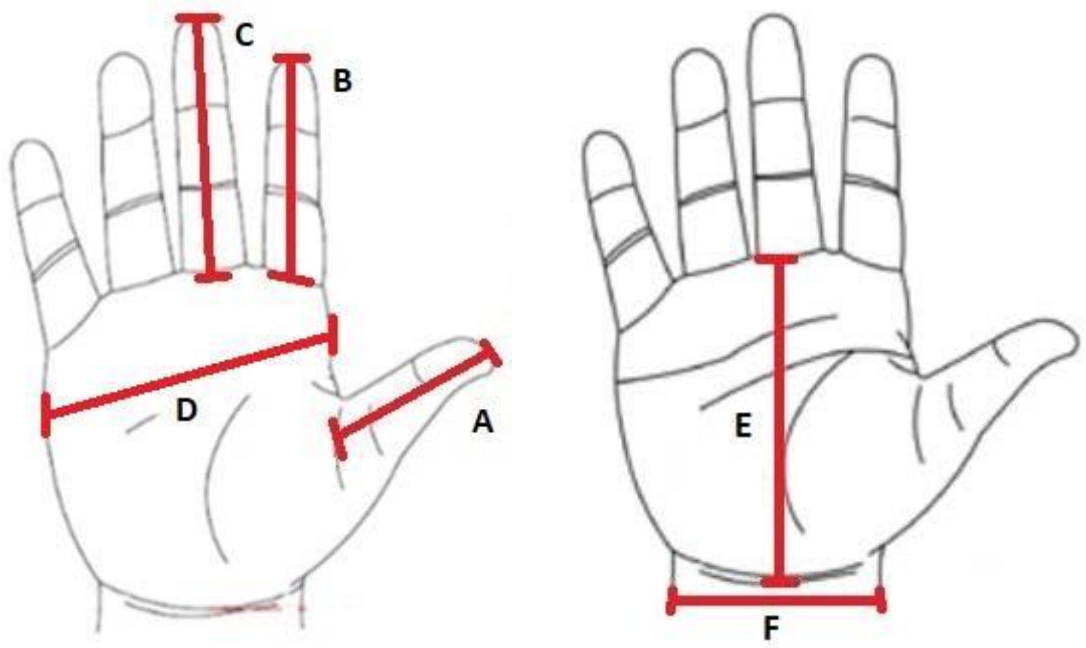

Con la finalidad de que mantenga un sustento estadístico con alta significancia para cada segmento definido en el estudio y diseño de los dedales. Para la toma de medidas se necesitó la ayuda de un calibrador Vernier de tipo analógico y una cinta de medición ergonómica. La base de datos utilizada para recolectar la información fue Microsoft Office Excel, para posteriormente ser procesados. Con un nivel de confianza del $95 \%$ y una precisión del 5\%, y con las medidas obtenidas fue posible determinar el percentil 5 y 95, necesarios para el diseño ergonómico de los dedales. Debido a las variaciones en las características antropométricas y morfológicas de cada persona, es muy importante realizar la toma de muestras a la población de interés o al área donde se desea implementar. Como se busca que los dedales tengan el mejor ajuste, también se creó un sistema de tallas, para la adecuación antropométrica. Con la información obtenida, se realizó el diseño en formato 3D con el software SolidWorks.

\section{Resultados y discusión}

Los resultados de las encuestas realizadas se observan en la figura 2. Se destaca que, el 87,5\% contestaron que han sufrido lesiones en las manos como callosidades, ampollas, irritación, rozaduras o incomodidad al realizar su actividad laboral, el $75 \%$ de las encuestadas 
respondieron que utilizan gasas como equipo de protección, el 64,5\% considera que el equipo de protección personal no es adecuado para las actividades que realizan y el 82,5\% opina que el producto propuesto sería de mucha ayuda para prevenir o disminuir las molestias que presentan, y además un 92,5\% estaría dispuesta a utilizarlo.

Figura 2. Resultado de la encuesta realizada a las trabajadoras de la empresa de ensamble de producto eléctrico.

1.- ¿Ha sufrido lesiones en las manos como incomodidad, irritación.

callosidades y/o ampollas al realizar su actividad laboral? 40 respuestas

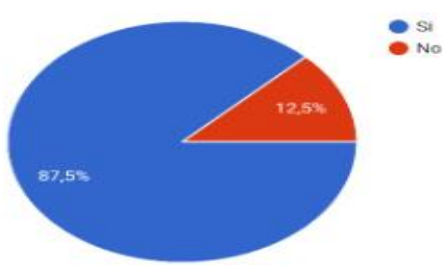

3.- ¿Considera que utilizar tres dedales reforzados en las áreas de los dedos que son más afectadas seria útil para desempeñar su trabajo?

40 respuestas

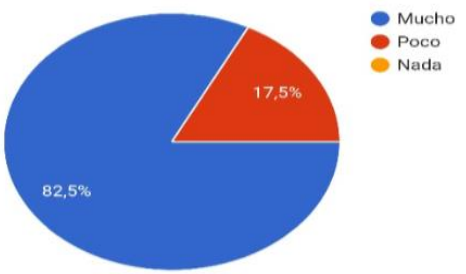

2.- ¿Utiliza gasas como equipo de protección personal para las manos?

40 respuestas

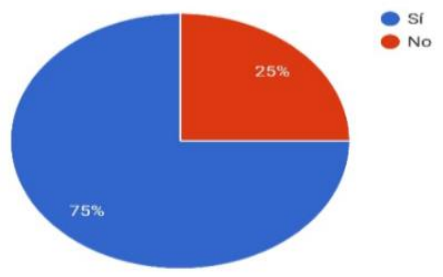

Si la respuesta anterior fue sí, ¿considera que es el adecuado? 31 respuestas

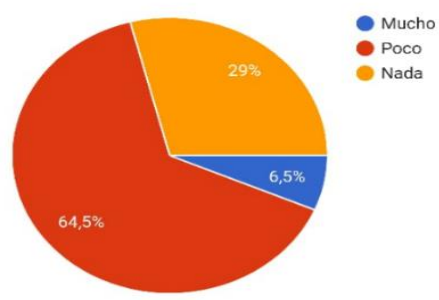

5.- ¿Utilizaria Thimblex en su trabajo?

40 respuestas

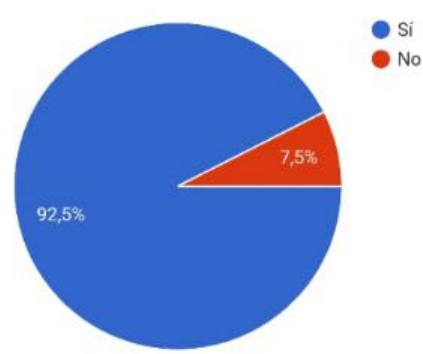

Se realizó el diseño del equipo de protección personal ergonómico, con ayuda de la información recopilada de las encuestas. Además, se confeccionó una tabla de las tallas basadas en los percentiles obtenidos.

Para la talla de los dedales se tomaron los códigos A y B, para la longitud de la mano se tomaron los códigos $\mathrm{C}$ y E, para la anchura de la mano se tomó el código $\mathrm{D}$, en ancho de la palma y por último el código $\mathrm{F}$ para determinar las medidas de la circunfernecia de la muñeca. 
Tabla 1. Medidas tomadas y resultados de percentiles.

\begin{tabular}{|c|c|c|c|}
\hline Código & Medida (cm) & Percentil 5 & Percentil 95 \\
\hline A & Largo del pulgar & $4,595 \mathrm{~cm}$ & $6,600 \mathrm{~cm}$ \\
\hline B & Largo del índice & $6,000 \mathrm{~cm}$ & $7,205 \mathrm{~cm}$ \\
\hline C & Largo del dedo medio & $6,595 \mathrm{~cm}$ & $7,905 \mathrm{~cm}$ \\
\hline D & Ancho de la palma & $8,795 \mathrm{~cm}$ & $10,32 \mathrm{~cm}$ \\
\hline E & Largo de la palma & $7,000 \mathrm{~cm}$ & $8,405 \mathrm{~cm}$ \\
\hline F & Circunferencia de la muñeca & $14,175 \mathrm{~cm}$ & $17,025 \mathrm{~cm}$ \\
\hline
\end{tabular}

Tabla 2. Tallas de acuerdo a los resultados obtenidos.

\begin{tabular}{|l|c|c|c|c|c|}
\hline \multicolumn{1}{|c|}{ Tallas } & S & M & L & XL & XXL \\
\hline Talla dedales & 6 & 7 & 7 & 8 & 8 \\
\hline Longitud de la mano & 11 & 13 & 12 & 14 & 16 \\
\hline Anchura de la mano & 7 & 7 & 8 & 8 & 9 \\
\hline Circunferencia de la muñeca & 13 & 14 & 15 & 16 & 17 \\
\hline
\end{tabular}

Nota. Medidas en cm.

Finalmente, se presenta el diseño del dedal para el uso de trabajadoras de la maquila en ensambles de componentes electrónicos. El cual consiste en básicamente un guante que proteja los dedos pulgar, índice y medio, que son los que más están expuestos a lesiones, además, contaría con un broche en la parte de la muñeca para que puedan usarlo eficientemente.

Figura 3. Dedal en diagonal.

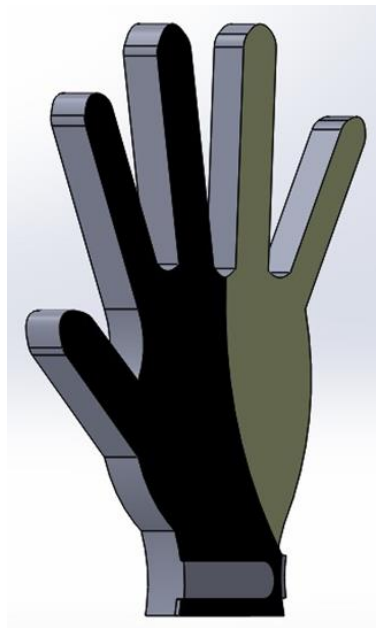

Figura 4. Dedal de frente.

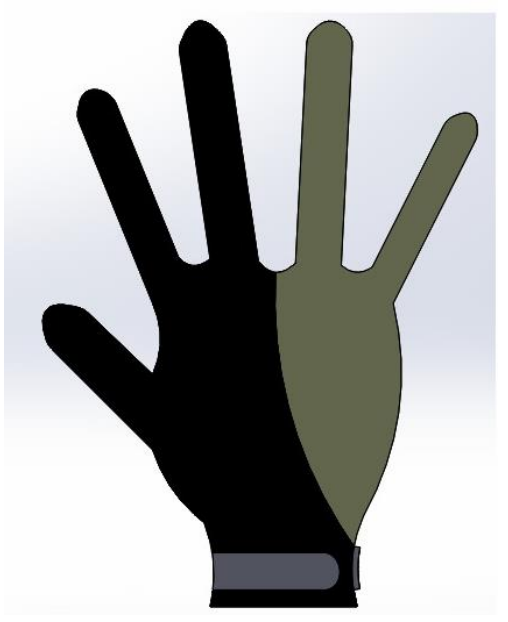

\section{Conclusiones}

Con los resultados obtenidos se proporcionó el diseño de un equipo de protección personal innovador, reiterando que con este se busca evitar incomodidad y/o lesiones en las trabajadoras, causadas por la fricción al momento de realizar sus actividades laborales, que requieren hacerse de forma repetitiva y por tiempos prolongados. Por lo que se cumplió con el objetivo, puesto que se pudo identificar con ayuda de una encuesta, la necesidad de un 
equipo de protección adecuado para las trabajadoras que ensamblan componentes electrónicos, además, con las medidas obtenidas se realizó la tabla de tallas, que va a brindar el mejor ajuste de los dedales a las trabajadoras, proporcionando no solo protección, sino que también funcionalidad y confort. Con ayuda de toda la información recabada, como segunda etapa, se presenta el producto físicamente, para que las trabajadoras prueben los dedales, y de esta forma comprobar o determinar la magnitud en la reducción de los riesgos físicos. 


\section{Referencias}

Alcántara, J. (2019, 23 de mayo). Buenas prácticas en la prevención de accidentes de manos. Marsh. $\quad$ https://www.marsh.com/pe/es/insights/marsh-college/buenas-practicas-en-laprevencion-de-accidentes-de-manos.html

Christopher \& Dana Reeve Foundation (2016, 16 de marzo). Cuidado de la piel. https://www.christopherreeve.org/es/international/top-paralysis-topics-in-spanish/skincare

Compañía de Producción BP, Administración de Seguridad y Salud Ocupacional, y Departamento de Seguros de Texas (2019, 9 de diciembre). Lesiones a los dedos, las manos, y muñecas. https://www.tdi.texas.gov/pubs/videoresourcessp/spt5fingerhandinjury.pdf

Martínez, M. del C., Fragiel, J., \& Nava N. R. (2003). Investigación de accidentes laborales con lesiones de las manos y los dedos. Salud de los trabajadores, 11(2), 99-113. https://dialnet.unirioja.es/servlet/articulo?codigo $=2938684$

NOTIMEX (2019, 12 de junio). Manos, las extremidades que más se dañan en accidentes laborales. 20 minutos. https://www.20minutos.com.mx/noticia/522554/o/manos-lasextremidades-que-mas-se-danan-en-accidentes-laborales/

Valle, M. L., \& Otero, M. (2015). Guía práctica de salud laboral para la valoración de: Aptitud en trabajadores con riesgo de exposición a carga física. Escuela Nacional de Medicina del Trabajo. $\quad$ http://gesdoc.isciii.es/gesdoccontroller?action=download\&id=25/01/2016400a0438b9

Wolfgang, L., \& Vedder, J. (2000). Capítulo 29: Ergonomía. En Enciclopedia de Salud y Seguridad en el Trabajo (pp. 1-110). Organización Internacional del Trabajo. https://www.insst.es/documents/94886/161958/Cap\%C3\%ADtulo+29.+Ergonom\%C3\%AD

$\underline{\mathrm{a}}$ 